\title{
A population study of the association between sexual function, sexual satisfaction and depressive symptoms in men
}

\author{
Alfredo Nicolosi ${ }^{\mathrm{a}, \mathrm{b}, *}$, Edson D. Moreira Jr. ${ }^{\mathrm{c}}$, Marco Villa ${ }^{\mathrm{a}}$, Dale B. Glasser ${ }^{\mathrm{d}}$ \\ ${ }^{a}$ Department of Epidemiology, Institute of Biomedical Technologies, National Research Council, Milan, Italy \\ ${ }^{\mathrm{b}}$ Gertrude H. Sergievsky Center, School of Public Health, Columbia University, New York, NY, USA \\ ${ }^{\mathrm{c}}$ Gonçalo Moniz Research Center, FIOCRUZ, Salvador, Brazil \\ ${ }^{\mathrm{d}}$ Pfizer Inc., New York, NY, USA
}

Received 10 March 2003; received in revised form 8 December 2003; accepted 8 December 2003

\begin{abstract}
Background: Depression and erectile dysfunction (ED) have a complex and bi-directional relationship. We examined the relationships between erectile dysfunction and depressive symptoms or diagnosed depression, sexual activity and sexual satisfaction.

Methods: A population survey of men aged 40-70 years was carried out in Brazil, Italy, Japan and Malaysia in $1997-1998$. A questionnaire was used to collect life style, sexual behaviors and medical data. Depressive symptoms were assessed using the Center for Epidemiologic Studies Depression Scale. ED was classified as moderate or complete if the men reported they were "sometimes" or "never" able to achieve and maintain an erection satisfactory for sexual intercourse. Only men with a sexual partner and not taking psychoactive drugs were considered.

Results: Diagnosed depression was reported by $2.0 \%$ of the men, depressive symptoms by $21.0 \%$. The prevalence of moderate or complete ED was $17.8 \%$. Sexual satisfaction related to the frequency of sexual intercourse and inversely related to depressive symptoms. Depressive symptoms were positively associated with being single (odds ratio [OR] 1.7), widowed, separated or divorced (OR 2.2), moderate or complete ED (1.8), heart disease (1.6) and smoking (1.6), and negatively associated with age, physical activity and frequency of sexual intercourse.

Limitations: Cross-sectional studies cannot establish a temporal cause-effect relationship. However, the confirmation of known associations reassures about the validity of the original findings.

Conclusions: The findings suggest that depressive symptoms are linked to ED by the mediation of decreased sexual activity and the dissatisfaction generated by the inability to have a healthy sexual life.
\end{abstract}

(C) 2004 Elsevier B.V. All rights reserved.

Keywords: Depressive symptoms; Depression; Erectile dysfunction; Population; Prevalence; Sexual activity

* Corresponding author. Department of Epidemiology and Medical Informatics, Institute of Biomedical Technologies, National Research Council, Via Fratelli Cervi 93, 20090 Segrate (Milan), Italy. Tel.: +39-0226422736; fax: +39-0226422770.

E-mail address: alfredo.nicolosi@itb.cnr.it (A. Nicolosi).

\section{Introduction}

Depression and sexual dysfunction are often reported as associated conditions in men. The direction of the association is unclear: does sexual dysfunction contribute to the onset of depression or is it depression 
that influences sexual function? Some researchers have included depression and affective disorders as important factors in the complex etiology of erectile dysfunction (ED) (Smith, 1998; Barlow, 1986; Jacobs et al., 1983), whereas others have stressed the role of ED in causing or exacerbating depressive symptoms (Shabsigh et al., 1998), and still others have pointed out that ED and depression may reinforce each other (Mathew and Weiman, 1962; Munjack et al., 1981; Nofzingzer et al., 1993). Almost all of these studies involved clinical series of patients with either ED or depression, and many did not use a control group.

Among the population studies of ED prevalence and risk factors, the Massachusetts Male Aging Study found that the prevalence of depressive symptoms - assessed using the Center for Epidemiologic Studies Depression rating scale (CES-D) - was linearly associated with the prevalence of ED (Araujo et al., 1998). The association between depressive symptoms and ED was also observed in a population study in Belgium (Mak et al., 2002). In the CrossNational Study of the Prevalence and Correlates of Erectile Dysfunction, we replicated these findings and also found an association between a history of diagnosed depression and ED (Nicolosi et al., 2003). A survey on the prevalence of concomitant ED and active depression among 199 outpatients seen at medical practices in Pennsylvania found a not statistically significant association (Kantor et al., 2002).

The possible role of ED as a risk factor for depression is suggested by the results of a few clinical trials. A German study of ED patients found that the frequency of self-reported depression (measured using the CES-D scale) was lower in the patients successfully treated with sildenafil than in those with untreated ED (Müller and Benkert, 2001). In a UK multicenter study, both the ED-specific Quality of Life Measure and the Hospital Anxiety and Depression Scale showed responsiveness to changes in ED before and after treatment (MacDonagh et al., 2002). These results suggest that ED is a risk factor for depression. On the other hand, the hypothesis that depression may be an important risk factor for erectile dysfunction is weakened by what was observed among the men of the Massachusetts Male Aging Study where, during the follow-up study, the presence of depressive symptoms at baseline was not a predictor of the incidence of ED (the odds ratio for the development of ED was 0.56 but not statistically significant) (Araujo et al., 2000).

We have conducted a cross-national survey of the prevalence of ED, concomitant medical and psychologic conditions, and behavioral factors in randomly selected population samples (Nicolosi et al., 2003), and would like to provide a contribution that may help to shed some light on the relationships between $\mathrm{ED}$ and depressive symptoms as mediated by sexual behavior and sexual satisfaction.

\section{Methods}

\subsection{Population}

The survey was carried out in Brazil, Italy, Japan and Malaysia (Nicolosi et al., 2003). In each country, a random sample of 2700 households was contacted and approximately 600 men aged 40-69 years were included in the final sample. The method of data collection varied on the basis of cultural considerations, being in-person interviews in Brazil, telephone interviews in Italy, self-completed questionnaires in Japan, and telephone and/or in-person interviews in Malaysia. The interview techniques were chosen with the aim of obtaining valid answers in the most suitable way for each country. The interviews were conducted between October 1997 and June 1998 using the same standardized questionnaire designed on the basis of suggestions coming from two focus groups held in each country. Each focus group was held with at least 30 men of the same age range of the target population. The group leader (one of the study's investigators) proposed each of the questionnaire's items to verify its acceptability, comprehension and correctness of phrasing. All items in the questionnaire had to be unanimously approved by the group. The questionnaire was translated, backtranslated and culturally adapted for each language, and collected information concerning the subjects' demographic, medical history, psychosocial profile, lifestyle habits and sexual behavior.

In order to study the relationship between sexual function, sexual activity and depressive status, the analysis was restricted to the men who reported having a stable sexual partner. Married men or men living with a woman were considered as not having a sexual 
partner if they did not report a sexual relationship with her. Also, since antidepressant drugs are suspected of affecting sexual function in men by inducing reduced sexual desire and arousal difficulties (Rosen et al., 1999; Ekselius and von Knorring, 2001), men taking these and other medications acting on the central nervous system were excluded in order to remove a possible cause of confounding.

\subsection{Definition of depressive symptoms, depression and use of psychoactive drugs}

The presence of depressive symptoms was assessed using the CES-D scale, which has been shown to be a valid screening tool for detecting groups at high risk of depression at population level (Weissman et al., 1977). We used a shortened scale with scores ranging from 5 to 20. The men whose scores fell into the upper quintile of the scale were classified as being affected by depressive symptoms. This cut-off was chosen with the conservative purpose of achieving a high specificity (other studies have used the upper quartile) (Mak et al., 2002). The main analysis of our study was based on depressive symptoms, but we also collected information about depression, as a complementary and confirmatory finding. The diagnosis of depression was based on self-reports: each of the subjects was asked whether he had ever been diagnosed as having depression by a physician. Two questions were asked about the use of drugs acting on the central nervous system. The first question, posed in conjunction with the question about ever having had a diagnosis of depression, asked whether the interviewed man was currently taking antidepressant medications. The second question asked whether the interviewed individual was currently using hypnotic, sedative or antidepressant drugs (which we for convenience, although improperly (Goodman and Gillman's, 2001), collectively called "psychoactive" drugs).

\subsection{Definition of $E D$}

On the basis of the United States National Institutes of Health and American Urological Association criteria (NIH Consensus Conference, 1993), ED was defined as "the persistent inability to achieve and/or maintain an erection sufficient for sexual activity". Each of our survey subjects was asked "How would you describe yourself?" and invited to choose one of four possible answers: "Always/Usually/Sometimes/Never able to get and keep an erection good enough for sexual intercourse". The subjects answering "Always" were classified as normal, whereas those answering "Usually" or "Sometimes" or "Never" were respectively classified as having "mild", "moderate" or "complete" ED. In the analysis, we conservatively focused on the men with "moderate" or "complete" ED, because the "mild" category may be a mix of men with intermittent situational problems or mild persistent ED.

\subsection{Definition of other characteristics and conditions}

The subjects were classified as being affected by a specific disease if they reported a physician's diagnosis or were taking medications for it. Prostate conditions included benign prostatic hyperplasia, a history of prostatitis, any prostate surgery and prostate cancer. Lower urinary tract symptoms were assessed by means of the International Prostate Symptoms Score (IPSS), and classified as absent or minor (IPSS $\leq 7$ ), moderate (8-19) or severe (20-35) (Barry et al., 1992). Smoking was defined as the smoking of cigarettes, cigars or a pipe, or the use of chewing tobacco, and the men were classified as non-smokers, former smokers or current smokers. Alcohol drinkers included all of the men who reported any drinking of wine, beer or spirits. The degree of physical activity was classified by the subjects as "below average", "average" or "above average" depending on the amount and frequency of physical activity at work and during leisure time. The men were asked whether they were satisfied with their sexual life and offered the choice of five answers: "extremely satisfied", "rather satisfied", "neither satisfied nor dissatisfied", "somewhat dissatisfied" and "dissatisfied". They were also asked whether the frequency of sexual intercourse with their partner corresponded to their desire, with the option to answer: "I have no desire", "as desired", "less than desired" and "higher than desired".

\subsection{Statistical analysis}

The replies to the completed questionnaires were double-entered into a computerized database to check for errors and analyze their internal consistency. De- 
pressive symptoms were analyzed (using the CES-D score) as a continuous variable in some analyses and as a dichotomous variable in the multivariate logistic analyis (with a cut-off point corresponding to the upper quintile).

The prevalences of all of the considered conditions were calculated by dividing the number of cases by the corresponding population. We used the chi square and the analysis of variance to test the difference between the categorical and continuous variables respectively (Fleiss, 1981; Neter and Wasserman, 1974). The strength of the association between variables was estimated using odds ratios (Schlesselman, 1982) (OR) and their 95\% confidence intervals (CI) (Woolf, 1955). Logistic regression analysis was used to estimate the association between depressive symptoms and the other variables, while adjusting for the simultaneous effect of all of the variables in the model (Cornfield, 1962).

\section{Results}

Of the 2417 men answering the question about ED, $1807(75 \%)$ reported that they currently had a sexual partner. Ninety-eight of these were currently taking anti-depressant drugs and were excluded from the analysis. Almost half of the 1709 studied men were in their $40 \mathrm{~s}$, and the great majority were married or living with their partners (Table 1). The level of education was rather high ( $46 \%$ of the men had gone to high school or had university degrees). The prevalence of medical conditions was that expected in men of this age. More than $90 \%$ of the men reported having had sexual intercourse in the previous 6 months, and nearly $80 \%$ of them were "rather" or "extremely" satisfied with their sexual life.

The prevalence of men reporting a diagnosis of depression was $2 \%$, but the prevalence of those presenting depressive symptoms (i.e. a CES-D score of $\geq 9$, corresponding to the upper quintile) was $21 \%$. The prevalence of diagnosed depression and that of depressive symptoms did not show statistically significant variations with age at the bivariate analyses (performed by contingency tables, analysis of variance and correlation).

Most of the men (55.3\%) aged 40-49 years reported they could "always" achieve and maintain
Table 1

Characteristics of the population of the cross-national study of the prevalence and correlates of erectile dysfunction (1997-1998), men with sexual partner and not taking psychoactive drugs

\begin{tabular}{|c|c|c|}
\hline $\begin{array}{l}\text { Characteristic } \\
\text { or condition }\end{array}$ & $\begin{array}{l}\text { Number } \\
\text { of men }\end{array}$ & $\begin{array}{l}\text { Proportion } \\
\text { of men }(\%)\end{array}$ \\
\hline \multicolumn{3}{|l|}{ Age group } \\
\hline $40-49$ & 846 & 49.5 \\
\hline $50-59$ & 515 & 30.1 \\
\hline $60-70$ & 348 & 20.4 \\
\hline \multicolumn{3}{|l|}{ Marital status } \\
\hline $\begin{array}{l}\text { Married or living } \\
\text { with partner }\end{array}$ & 1564 & 91.6 \\
\hline Single & 57 & 3.3 \\
\hline $\begin{array}{l}\text { Widow, separated, } \\
\text { divorced }\end{array}$ & 87 & 5.1 \\
\hline \multicolumn{3}{|l|}{ Education } \\
\hline High school not completed & 914 & 53.5 \\
\hline High school completed & 618 & 36.2 \\
\hline Univeristy degree & 176 & 10.3 \\
\hline Currently employed & 1272 & 74.5 \\
\hline Diabetes & 139 & 8.1 \\
\hline Hypertension & 358 & 21.0 \\
\hline Heart disease & 119 & 7.0 \\
\hline Ulcer & 161 & 9.4 \\
\hline Prostate diseases & 124 & 7.3 \\
\hline \multicolumn{3}{|l|}{$\begin{array}{l}\text { Lower urinary tract } \\
\text { symptoms }\end{array}$} \\
\hline Absent or minor & 1435 & 86.2 \\
\hline Moderate & 198 & 11.9 \\
\hline Severe & 32 & 1.9 \\
\hline Diagnosed depression & 34 & 2.0 \\
\hline Depression-CES & 347 & 21.0 \\
\hline \multicolumn{3}{|l|}{ Erectile dysfunction } \\
\hline No & 1405 & 82.2 \\
\hline Moderate & 265 & 15.5 \\
\hline Complete & 39 & 2.3 \\
\hline \multicolumn{3}{|l|}{ Physical activity } \\
\hline Less than average & 130 & 7.6 \\
\hline Average & 341 & 20.0 \\
\hline More than average & 1238 & 72.4 \\
\hline Current smoker & 635 & 37.6 \\
\hline Current drinker & 937 & 54.8 \\
\hline $\begin{array}{l}\text { Intercourse during } \\
\text { last } 6 \text { months }\end{array}$ & 1569 & 93.2 \\
\hline \multicolumn{3}{|l|}{ Sexually satisfied } \\
\hline Extremely & 528 & 31.0 \\
\hline Rather & 834 & 48.9 \\
\hline Average & 232 & 13.6 \\
\hline Little & 82 & 4.8 \\
\hline Not at all & 28 & 1.6 \\
\hline
\end{tabular}

an erection sufficient for satisfactory sexual intercourse; after the age of 50 , this proportion began to decline, and reached $22.1 \%$ among the men aged $>60$ 
Table 2

Mean monthly number of episodes of sexual intercourse and degree of satisfaction with sexual life, by age group

\begin{tabular}{lcclll}
\hline $\begin{array}{l}\text { Age group } \\
\text { (years) }\end{array}$ & Extremely satisfied & Rather satisfied & $\begin{array}{l}\text { Neither satisfied } \\
\text { nor unsatisfied }\end{array}$ & $\begin{array}{l}\text { Somewhat } \\
\text { dissatisfied }\end{array}$ & Dissatisfied \\
\hline $40-49$ & $12.7 \pm 8.9(318)$ & $10.0 \pm 5.6(379)$ & $7.4 \pm 6.7(103)$ & $7.1 \pm 6.5(39)$ & $5.7 \pm 8.6(7)$ \\
$50-59$ & $10.2 \pm 8.8(135)$ & $7.4 \pm 4.2(275)$ & $5.0 \pm 4.3(66)$ & $4.8 \pm 4.6(26)$ & $3.0 \pm 6.1(11)$ \\
$60-70$ & $7.5 \pm 5.6(75)$ & $5.1 \pm 3.6(180)$ & $2.9 \pm 3.4(63)$ & $2.6 \pm 4.1(17)$ & $1.9 \pm 2.7(10)$ \\
\hline
\end{tabular}

Values are mean \pm standard deviation; in parenthesis, number of men.

years. The proportion of men who could "sometimes" achieve and maintain an erection sufficient for satisfactory sexual intercourse was $9.1 \%$ in the $40-49$ year age group, $16.3 \%$ in the $50-59$ year age group, and $29.9 \%$ in the $60-69$ year age group. None of the men in the youngest age group could "never" achieve and maintain an erection, but the corresponding figures in the other age groups were $1.2 \%$ and $9.5 \%$.

In order to investigate the possible association between ED and depression, we first considered the relationship between the frequency of sexual intercourse and the degree of satisfaction with sexual life (Table 2). These were correlated in all age groups: in the youngest age group, the mean number of episodes of sexual intercourse per month was 12.7 among the "extremely satisfied" men and 5.7 among the "dissatisfied" men; the corresponding figures in the oldest age group were 7.5 and 1.9 (the differences were statistically significant: $p<0.0001)$.
The mean number of episodes of sexual intercourse per month reported by the men with moderate or complete ED was lower than that reported by the men without ED (33\% lower in the youngest and $57 \%$ lower in the oldest age group) (Fig. 1). When asked whether the frequency of sexual intercourse with their partner corresponded to their desire, $49.3 \%$ of the men with and $75.7 \%$ of those without ED answered that it did, and $29.7 \%$ and $15.9 \%$ declared that it was less than desired $(14.9 \%$ of the men with and $1.6 \%$ of those without ED reported no sexual desire, whereas $6.1 \%$ and $6.7 \%$ complained that the frequency of sexual intercourse was more than desired).

The prevalence of depressive symptoms measured by the CES-D scores linearly correlated with the degree of sexual satisfaction. The mean CES-D score was 6.6 (standard deviation [SD] 2.0) among the extremely satisfied men, 7.0 (SD 2.3) among the

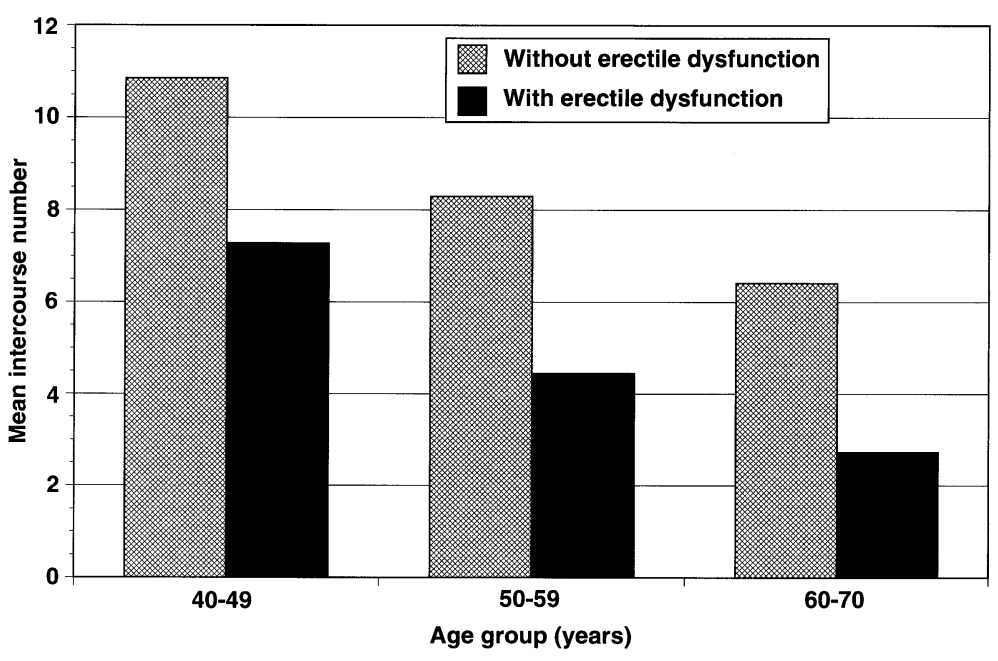

Fig. 1. Mean number of episodes of sexual intercourse per month reported by men with and without moderate or complete erectile dysfunction, by age group. 


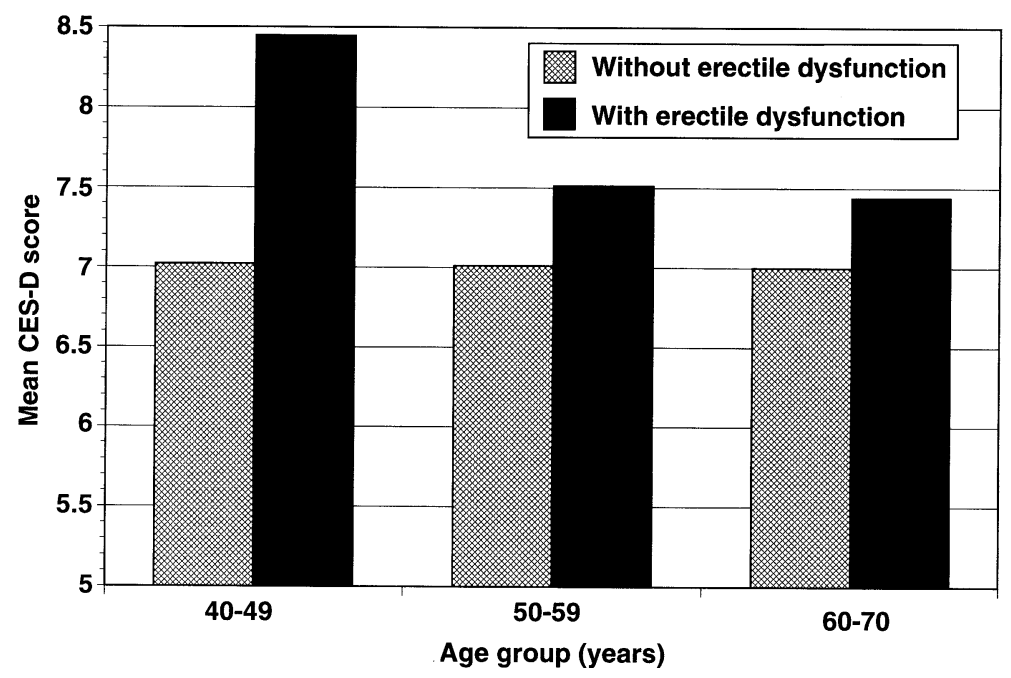

Fig. 2. Mean CES-D score in men with and without moderate or complete erectile dysfunction, by age group.

rather satisfied men, 7.9 (SD 2.7) among the men who were neither satisfied nor dissatisfied, 9.1 (SD 3.3) among the somewhat dissatisfied men and 10.2 (SD 4.1) among the men who were dissatisfied. The differences between these groups was statistically significant with a $p$ value of $<0.0001$.

Examinination of the mean CES-D scores by age group and ED status showed that the prevalence of depressive symptoms was higher in the men with ED (Fig. 2). The differences in the CES-D scores between the men with and without ED was greater at younger ages: $41.3 \%$ among those aged $40-49$ years, $19.9 \%$ among those aged $50-59$ years, and $18.0 \%$ among those aged 60-69 years $(p<0.0001)$.

\subsection{Diagnosed depression}

Thirty-four men $(2.0 \%)$ reported that they had been diagnosed as having depression and were not currently taking anti-depressive drugs. There were $23(1.6 \%)$ men with depression among the 1405 without ED, and 11 (3.6\%) among the 304 with ED. The odds ratio for depression was 2.3 (95\% CI: 1.1, 4.7) among men with moderate or complete ED in comparison with the men without ED.

Out of the 34 men who reported to ever having received a diagnosis of depression, 16 presented with depressive symptoms (a CES-D score $\geq 9$ ) at the interview.

\subsection{Factors associated with depressive symptoms}

We investigated the association of depressive symptoms (a CES-D score $\geq 9$ ) with ED while controlling for the presence of other possibly associated factors and adjusting by country, using a logistic regression model (Table 3). Depressive symptoms resulted associated with age at this multivariate analysis, with lower

Table 3

Associations between depressive symptoms and patients' characteristics (logistic regression analysis adjusted by country)

\begin{tabular}{lll}
\hline Characteristic or condition & $\begin{array}{l}\text { Odds } \\
\text { ratio }\end{array}$ & $\begin{array}{l}95 \% \text { Confidence } \\
\text { interval }\end{array}$ \\
\hline $\begin{array}{l}\text { Age group (years) } \\
40-49\end{array}$ & 1 & $\begin{array}{l}\text { (reference) } \\
50-59\end{array}$ \\
$\quad 0.70$ & $0.52-0.95$ \\
$\quad 0-70$ & 0.44 & $0.30-0.66$ \\
$\quad$ Marital status & & \\
$\quad$ Married or living with & 1 & (reference) \\
$\quad$ partner & & \\
$\quad$ Single & 1.75 & $0.94-3.28$ \\
$\quad$ Widower, separated, & 2.17 & $1.32-3.59$ \\
$\quad$ divorced & & \\
Erectile dysfunction & 1.81 & $1.28-2.55$ \\
$\quad$ (moderate or complete) & & \\
$\quad$ Heart disease & 1.58 & $1.01-2.50$ \\
Current smoker & 1.62 & $1.25-2.10$ \\
Physically active & 0.52 & $0.34-0.80$ \\
Frequency of intercourse & 0.59 & $0.43-0.80$ \\
$\quad(\geq 5$ per month) & & \\
\hline
\end{tabular}


odds ratios in elderly men. There was an association between being single (OR 1.75) or widowed, separated or divorced men (OR 2.17). A positive association was also found with moderate or complete ED (1.81), heart disease (1.58) and smoking (1.62). There was an inverse association with the degree of physical activity (a $48 \%$ lower risk among the most active category) and the monthly frequency of sexual intercourse.

\section{Discussion}

The aim of this study was to determine whether sexual function, sexual satisfaction and depressive symptoms are inter-related. We studied random population samples from four different countries with very different socioeconomic and cultural backgrounds, factors which can influence the prevalence of depression (Gilman et al., 2002), and were controlled in the analysis. The international context is a major element of the originality of this study because diversity, if controlled, adds to the validity of the results.

We considered only men who reported to have a stable sexual partner and not taking psychoactive medications in order to focus on the relationship between sexual function and depressive symptoms and avoid confounding bias. It is certain that crosssectional studies can suggest but not establish a temporal, cause-effect relationship. Nonetheless, we think that the results of this study are quite convincing in demonstrating an association between ED and depression.

The frequency of sexual intercourse was associated with sexual satisfaction in our population, and it is reasonable to interpret this result as meaning that sexual satisfaction is a consequence rather than an antecedent of sexual activity. We also found that the prevalence of depressive symptoms inversely correlated with the degree of sexual satisfaction. The association of sexual satisfaction with depressive symptoms had a linear trend suggesting a "dose-response" relationship (i.e. the less the satisfaction, the more severe the depressive symptoms, and vice versa).

If these associations are true, the potential influence of ED on depressive symptoms is a consequence. The frequency of sexual activity among men with ED is lower than that among men without ED, a finding that has also been documented elsewhere (Koskimaki et al., 2000). Although a proportion of men with ED seems to cope with this limitation by adjusting their expectations $(15 \%$ of our respondents with ED reported no desire for sexual intercourse with their partners), the majority experience fewer episodes of sexual intercourse than desired. This situation generates dissatisfaction with their sexual life, a possible sense of frustration, and may eventually lead to the development of depressive symptoms. This conclusion is supported by our observation that, among the men with ED, the prevalence of depressive symptoms was higher among younger men; i.e. those with a greater expectation of a normal and healthy sexual life.

The multivariate analysis, in which the independent associations between depressive symptoms and each variable were studied while controlling for the presence of concomitant risk factors and the country of residence, confirmed that ED is a predictor of a depressed status in men. In the course of the analysis, different models have been elaborated. In a model which did not include the frequency of sexual intercourse, the odds ratio of ED was higher than that reported in Table 3, because ED is associated with a lower frequency of sexual intercourse. The fact that ED is correlated with depressive symptoms even after controlling for the frequency of sexual intercourse suggests that this condition has a deeper impact on men's psychological status than just its physical consequences.

Depressive symptoms showed an association with age in the logistic regression, in contrast to the bivariate analysis. The age prevalence of depression and depressive symptoms is an open research topic, more in women than in men (Bebbington et al., 1988). Among men, it seems that two peaks exist in the prevalence of major depression, one in late old age and the other in middle age or earlier (Snowdon, 2001). Self-reported depression symptoms show the highest prevalence among the youngest and the very old ( $>75$ years) (Karel, 1997; Molarius and Janson, 2002; Daradkeh et al., 2002). The negative association with age that we found in our study was the result of a multivariate model which considered other concomitant factors besides age. Also, the prevalence of depression in this study is not representative of the population since more than one half of the men with a history of diagnosis of depression was removed from the analysis because they reported the use of antidepressant drugs. 
The development of depressive symptoms may be due to many reasons, and we by no means want to suggest that ED is its main causal factor. Family, social and environmental factors, health status, and even genetic factors may all be involved. The influence of heart disease on depression and ED has been demonstrated in other studies (Goldstein, 2000; Roose and Seidman, 2000). Smoking and low levels of physical activity have been described as being more frequent among depressed than non-depressed subjects (Frerichs et al., 1981; Giovino et al., 1995; Allgower et al., 2001; Strawbridge et al., 2002). Both factors are also known to be associated with ED (Bacon et al., 2001). The psychological support provided by the family has been described in a number of studies (Robins and Regier, 1991) showing that people who have never been married and those who have experienced bereavement are at special risk for depression. The fact that our study confirms previously known associations is reassuring about the validity of its original findings.

In conclusion, the association between depressive symptoms and impaired sexual function has been previously observed. The originality of our study lies in the fact that it was based on a random population sample drawn from different countries, and in that we were able to demonstrate that depressive syptoms are linked to ED by the mediation of decreased sexual activity and the dissatisfaction generated by the inability to have a healthy sexual life. This suggests a causal association. Since these inferences were drawn from a cross-sectional study, further research is needed to confirm the temporal sequence between ED and depressive symptoms.

\section{Acknowledgements}

This work was partially funded by an unconditional grant from Pfizer.

\section{References}

Allgower, A., Wardle, J., Steptoe, A., 2001. Depressive symptoms, social support, and personal health behaviors in young men and women. Health Psychol. 20, 223-227.

Araujo, A.B., Durante, R., Feldman, H.A., Goldstein, I., Mckinlay, J.B., 1998. The relationship between depressive symptoms and male erectile dysfunction: cross-sectional results from the Massachusetts Male Aging Study. Psychosom. Med. 60, 458-465.

Araujo, A.B., Johannes, C.B., Feldman, H.A., Derby, C.A., McKinley, J.B., 2000. Relation between psychosocial risk factors and incident erectile dysfunction: prospective results from the Massachusetts male aging study. Am. J. Epidemiol. 152, $533-541$.

Bacon, C., Mittleman, M., Glasser, D., Rimm, E., 2001. Can lifestyle factors help prevent erectile dysfunction? Eur. Urol. 39 (Suppl. 5), 17.

Barlow, D.H., 1986. Causes of sexual dysfunction: the role of anxiety and cognitive interference. J. Consult. Clin. Psychol. 54, $140-148$.

Barry, M.J., Fowler Jr., F.J., O’Leary, M.P., et al., 1992. The American urological association symptom index for benign prostatic hyperplasia. J. Urol. 148, 1549-1557.

Bebbington, P.E., Dunn, G., Jenkins, R. et al., 1988. The influence of age and sex on the prevalence of depressive conditions:report from the national survey of psychiatric morbidity. Psychol. Med. 28, 9-19.

Cornfield, J., 1962. Joint dependence of the risk of coronary heart disease on serum cholesterol and systolic blood pressure: a discriminant function analysis. Fed. Proc. 21, 58-61.

Daradkeh, T.K., Ghubash, R., Abou-Saleh, M.T., 2002. Al Ain community survey of psychiatric morbidity: II. Sex differences in the prevalence of depressive disorders. J. Affect. Disord. 72, $167-176$.

Ekselius, L., von Knorring, L., 2001. Effect on sexual function of long-term treatment with selective serotonin reuptake inhibitors in depressed patients treated in primary care. J. Clin. Psychopharmacol. 21, 154-160.

Fleiss, J.L., 1981. Statistical Methods for Rates and Proportions, 2nd ed. Wiley, New York.

Frerichs, R.R., Aneshensel, C.S., Clark, B.A., et al., 1981. Smoking and depression: a community survey. Am. J. Public Health 71, $637-640$.

Gilman, S.E., Kawachi, I., Fitzmaurice, G.M., Buka, S.L., 2002. Socioeconomic status in childhood and the lifetime risk of major depression. Int. J. Epidemiol. 31, 359-367.

Giovino, G.A., Henningfield, J.E., Tomar, S.L., Escobedo, L.G., Slade, J., 1995. Epidemiology of tobacco use and dependence. Epidemiol. Rev. 17, 48-65.

Goldstein, I., 2000. The mutually reinforcing triad of depressive symptoms, cardiovascular disease, and erectile dysfunction. Am. J. Cardiol. 86, 41F-45F (suppl).

Goodman, L.S., Gillman, A., 2001. Goodman and Gillman's-The Pharmacologial Basis of Therapeutics, 10th ed. McGraw-Hill, New York.

Jacobs, J.A., Fishkin, R., Cohen, S., Goldman, A., Mulholland, S.G., 1983. A multisciplinary approach to the evaluation and management of male sexual dysfunction. J. Urol. 129, 35-37.

Kantor, J., Bilker, W.B., Glasser, D.B., Margolis, D.J., 2002. Prevalence of erectile dysfunction and active depression: an analytic cross-sectional study of general medical patients. Am. J. Epidemiol. 156, 1035-1042.

Karel, M.J., 1997. Aging and depression: vulnerability and stress across adulthood. Clin. Psychol. Rev. 17, 847-879. 
Koskimaki, J., Hakama, M., Huhtala, H., Tammela, T.L.J., 2000. Effect of erectile dysfunction on frequency of intercourse: a population based prevalence study in Finland. J. Urol., $367-370$.

MacDonagh, R., Ewings, P., Porter, T., 2002. The effect of erectile dysfunction on quality of life: psychometric testings of a new quality of life measure for patients with erectile dysfunction. J. Urol. 167, 212-217.

Mak, R., De Backer, G., Kornitzer, M., De Meyer, J.M., 2002. Prevalence and correlates of erectile dysfunction in a population-based study in Belgium. Eur. Urol. 41, 132-138.

Mathew, R.J., Weiman, M.L., 1962. Sexual dysfunctions in depression. Arch. Sex. Behav. 11, 323-328.

Molarius, A., Janson, S., 2002. Self-rated health, chronic diseases, and symptoms among middle-aged and elderly men and women. J. Clin. Epidemiol. 55, 364-370.

Müller, M.J., Benkert, O., 2001. Lower self-reported depression in patients with erectile dysfuncion after treatment with sildenafil. J. Affect. Disord. 66, 255-261.

Munjack, D.J., Oziel, L.J., Kanno, P.H., Whipple, K., Leonard, M.D., 1981. Psychological characteristics of males with secondary erectile failure. Arch. Sex. Behav. 10, 123-131.

Neter, J., Wasserman, W., 1974. Applied Linear Statistical Models. Richard D. Irwin, Homewood, IL.

Nicolosi, A., Moreira, E.D., Shirai, M., Tambi, M.I.B., Glasser, D.B., 2003. Epidemiology of erectile dysfunction in four countries: cross-national study of the prevalence and correlates of erectile dysfunction. Urology 61, 201-206.

NIH Consensus Conference, 1993. Impotence. NIH Consensus Development Panel on Impotence. JAMA 270, 83-90.
Nofzingzer, E.A., Thase, M.E., Reynolds, C.F., et al., 1993. Sexual function in depressed men. Arch. Gen. Psychiatry 50, 24-30.

Robins, L.N., Regier, D.A., 1991. Psychiatric Disorders in America: The Epidemiologic Catchment Area Study. The Free Press, New York, NY.

Roose, S.P., Seidman, S.N., 2000. Sexual activity and cardiac risk: is depression a contributing factor? Am. J. Cardiol. 86, 38F-40F (suppl).

Rosen, R.C., Lane, R.M., Menza, M., 1999. Effects of SSRIs on sexual function: A Critical Review. J. Clin. Psychopharmacol. $19,67-85$.

Schlesselman, J.J., 1982. Case-control Studies: Design, Conduct, Analysis. Oxford Univ. Press, New York, pp. 227-290.

Shabsigh, R., Klein, L.T., Seidman, S., Kaplan, S.A., Lehrhoff, B.J., Ritter, J., 1998. Increased incidence of depressive symptoms in men with erectile dysfunction. Urology 52, 848-852.

Smith, A.D., 1998. Psychologic factors in the multidisciplinary evaluation and treatment of erectile dysfunction. Urol. Clin. North Am. 15, 41-51.

Snowdon, J., 2001. Is depression more prevalent in old age? Aust. N. Z. J. Psychiatry $35,782-787$.

Strawbridge, W.J., Deleger, S., Roberts, R.E., Kaplan, G.A., 2002. Physical activity reduces the risk of subsequent depression for older adults. Am. J. Epidemiol. 156, 328-334.

Weissman, M.M., Sholomskas, D., Pottenger, M., Prusoff, B.A., Locke, B.Z., 1977. Assessing depressive symptoms in five psychiatric populations: a validation study. Am. J. Epidemiol. 106, $203-214$.

Woolf, B., 1955. On estimating the relation between blood group and disease. Ann. Hum. Genet. 19, 251-253. 